\title{
Surface plasmon-driven second-harmonic generation asymmetry in anisotropic plasmonic crystals
}

\author{
A. L. Chekhov, ${ }^{1,{ }^{*}}$ I. Razdolski, ${ }^{2, \dagger}$ A. Kirilyuk, ${ }^{2}$ Th. Rasing, ${ }^{2}$ A. I. Stognij, ${ }^{3}$ and T. V. Murzina ${ }^{1}$ \\ ${ }^{1}$ Department of Physics, Moscow State University, 119991 Moscow, Russia \\ ${ }^{2}$ Radboud University, Institute for Molecules and Materials, 6525 AJ Nijmegen, The Netherlands \\ ${ }^{3}$ Scientific-Practical Materials Research Centre of NAS of Belarus, 220072 Minsk, Belarus \\ (Received 22 January 2016; revised manuscript received 25 March 2016; published 11 April 2016)
}

\begin{abstract}
We report a strong angular asymmetry of optical second-harmonic generation (SHG) in plasmonic crystals formed by an Au grating on top of an anisotropic bismuth-thulium iron garnet (BTIG) film. We found that a weak anisotropy-driven angular SHG asymmetry of the crystalline BTIG is resonantly enhanced and reaches $95 \%$ as the surface plasmon polariton (SPP) is excited at the Au/BTIG interface. The asymmetry introduced by an interference of the odd and even (with respect to the angle of incidence) SHG fields is attributed to the anisotropy of the BTIG film. The angular SHG spectra are reproduced using a simple model with a resonant SPP-induced SHG contribution from the anisotropic Au/BTIG interface. The observed asymmetry of the SHG provides valuable insight into the mechanisms of nonlinear plasmonics and can noticeably expand its abilities regarding active light conversion and manipulation.
\end{abstract}

DOI: 10.1103/PhysRevB.93.161405

Modern approaches in nano-optics and photonics extensively utilize surface plasmon polaritons (SPPs) as a natural tool for efficient light-matter interaction [1-10]. The plasmondriven localization of the electromagnetic field facilitates an enhancement of the nonlinear-optical processes. Since the pioneering works on second-harmonic generation (SHG) at surface plasma resonances [11,12], a plethora of nonlinear-optical effects have been successfully enhanced with SPPs [13-20]. As compared to linear optics, nonlinear plasmonics [21] expands the variety of SPP excitation conditions, thus opening up more options for the efficient coupling of light to plasmonic media.

The remarkable progress made over the years indicates the complex nature of the SPP-induced nonlinear-optical response beyond the local electromagnetic field enhancement. For instance, the latter is accompanied by a significant phase shift of the second-harmonic response [22-24], which can be unambiguously detected in interference experiments. An interplay of the resonant phase shift with the interference of multiple nonlinear-optical sources can lead to a complex behavior of the SHG output in the vicinity of the SPP resonance [15,16,25]. Further, a new level of control over the SHG in plasmonic structures can be achieved in systems with broken symmetry [16,26]. As such, new effects in nonlinear plasmonics can be expected, when SPPs are excited at anisotropic metal/dielectric interfaces [27].

In this Rapid Communication, we analyze a showcase of such systems, being a periodic Au plasmonic grating on top of an anisotropic (Bi, Tm) ${ }_{3} \mathrm{Fe}_{5} \mathrm{O}_{12}$ (BTIG) (210) film. Here the total SHG output is given by the interference of the SHG produced at both interfaces of the Au grating and is strongly modulated by the SPP excitation. We show that upon reversing the angle of incidence and changing the SPP propagation direction, a nearly $95 \%$ modulation of SHG intensity can be achieved. This large modulation is understood in terms of an

\footnotetext{
*chekhov@shg.ru

${ }^{\dagger}$ Present address: Physical Chemistry Dept., Fritz Haber Institute of Max Planck Society, Faradayweg 4-6, 14195 Berlin, Germany.
}

interference between the competing SHG fields generated at two different interfaces and possessing different parity with respect to the angle of incidence. The existence of such terms is provided by the anisotropy of the BTIG film. We also discuss the differences between the bare BTIG film exhibiting only a weak asymmetry and the plasmonic crystal with the strong SPP-driven electric field localization at the Au/BTIG interface. Our results have important implications for the field of nonlinear plasmonics, illustrating the SPP-induced SHG response of anisotropic interfaces.

A schematic of the experiment is sketched in Fig. 1. Fundamental radiation (Ti:sapphire laser $50 \mathrm{~mW}$ average output, $100 \mathrm{fs}$ pulse duration, $80 \mathrm{MHz}$ repetition rate) in the spectral range of 740-865 nm excites SPP resonances at either air/Au or Au/BTIG interface. The $x$ axis is parallel to the [001] crystallographic direction of the BTIG film. Transmitted SHG intensity is measured for the $p-\omega, p-2 \omega$ combination of polarizations versus the fundamental wavelength and angle of incidence. The latter was swept from $-30^{\circ}$ to $+30^{\circ}$ in order to address the symmetry of the SHG response with respect to the SPP propagation direction. The system under study is similar to those used in a number of recent experiments [5,20,24,28-31] and is known to demonstrate effective SPP excitation at both interfaces in the visible and near infrared spectral range. The measurements were performed on a nonmagnetized BTIG film, and the magnetization-induced effects were averaged over the domain structure.

Figure 2(a) shows the transmission spectra of the sample. Here, solid and dashed lines correspond to the dispersion of $\mathrm{Au} / \mathrm{BTIG}$ and air/Au SPPs, respectively, given by the wellknown dispersion relations [32]:

$$
k_{\mathrm{SPP}}=\frac{2 \pi}{\lambda} \sqrt{\frac{\varepsilon_{\mathrm{Au}} \varepsilon_{\mathrm{d}}}{\varepsilon_{\mathrm{Au}}+\varepsilon_{\mathrm{d}}}}=\frac{2 \pi}{\lambda} \sin \theta+m \frac{2 \pi}{d},
$$

where $\lambda$ is the wavelength of light in vacuum, $k_{\text {SPP }}$ the SPP wave vector, $\varepsilon_{\mathrm{Au}}, \varepsilon_{\mathrm{d}}$ the permittivities of $\mathrm{Au}$ and the dielectric (either BTIG or air), $m$ an integer, and $d$ the grating period. Sharp and narrow parallel features in the transmittance spectra correspond to the excitation of waveguide modes $[30,33]$ 


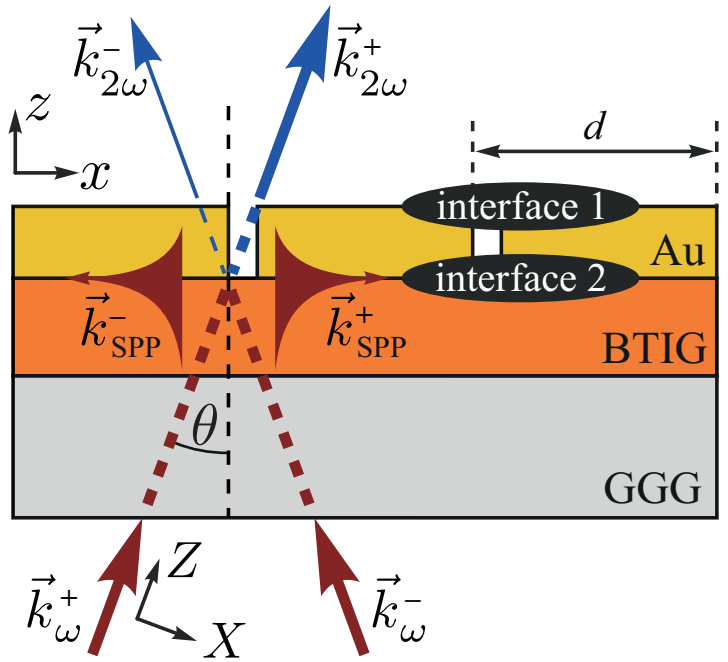

FIG. 1. Scheme of a 1D plasmonic crystal consisting of a gold grating formed on top of a bismuth-tulium iron garnet layer, grown epitaxially on a gallium gadolinium garnet substrate. The grating period $d=850 \mathrm{~nm}$ and the air gap width is $100 \mathrm{~nm}$. (XOZ) corresponds to the plane of incidence. SHG intensity measured in transmission geometry strongly differs for the opposite angles of incidence.

in a thin $(2.2 \mu \mathrm{m})$ BTIG film. These modes can be easily distinguished from the SPPs due to their different dispersion relations. The transmission spectra reveal Fano-type resonances, similar to those observed previously in plasmonic crystals $[5,29-31,34,35]$. The transmission was found to be symmetric with respect to the angle of incidence $\theta$ within $3 \%$ accuracy.

In contrast, strong asymmetry was found in the SHG intensity spectra [Fig. 2(b)]. It is seen that the SHG spectrum is highly asymmetric with respect to the angle of incidence $\theta$; that is, the SHG intensity differs significantly when being measured for the angles $\theta$ and $-\theta$ (Fig. 1). The asymmetry is clearly enhanced in the vicinity of the SPPs at the Au/BTIG interface (solid lines), but not the air/Au ones (dashed lines). This is an indication that in the latter case the SHG is enhanced due to the SPP fundamental field localization at the outer air/Au interface (interface 1 in Fig. 1), which acts as the main nonlinear source. However, when the SPP is excited at the $\mathrm{Au} / \mathrm{BTIG}$ interface, redistribution of the electromagnetic field changes the relative efficiency of the SHG generated at the two interfaces outlined in Fig. 1, and their interference results in a complex spectral shape of the SHG response. Further, the largest SHG signal is detected in the vicinity of the intersection of Au/BTIG plasmons with the waveguide modes at positive $\theta$ angles. Measurements at the negative angles in the same spectral and angular ranges yield considerably smaller signals, again producing the SHG asymmetry.

A strong affinity of this asymmetry effect for the SPP resonance is clearly demonstrated by the angular cross section of the SHG spectrum for the $850 \mathrm{~nm}$ fundamental wavelength [Fig. 3(a)]. Here, the Au/BTIG SPP resonance is isolated from the other excitations in the system. A double minimum feature in the SHG spectrum, observed for the positive $\theta$ angles and associated with the Au/BTIG plasmon resonance

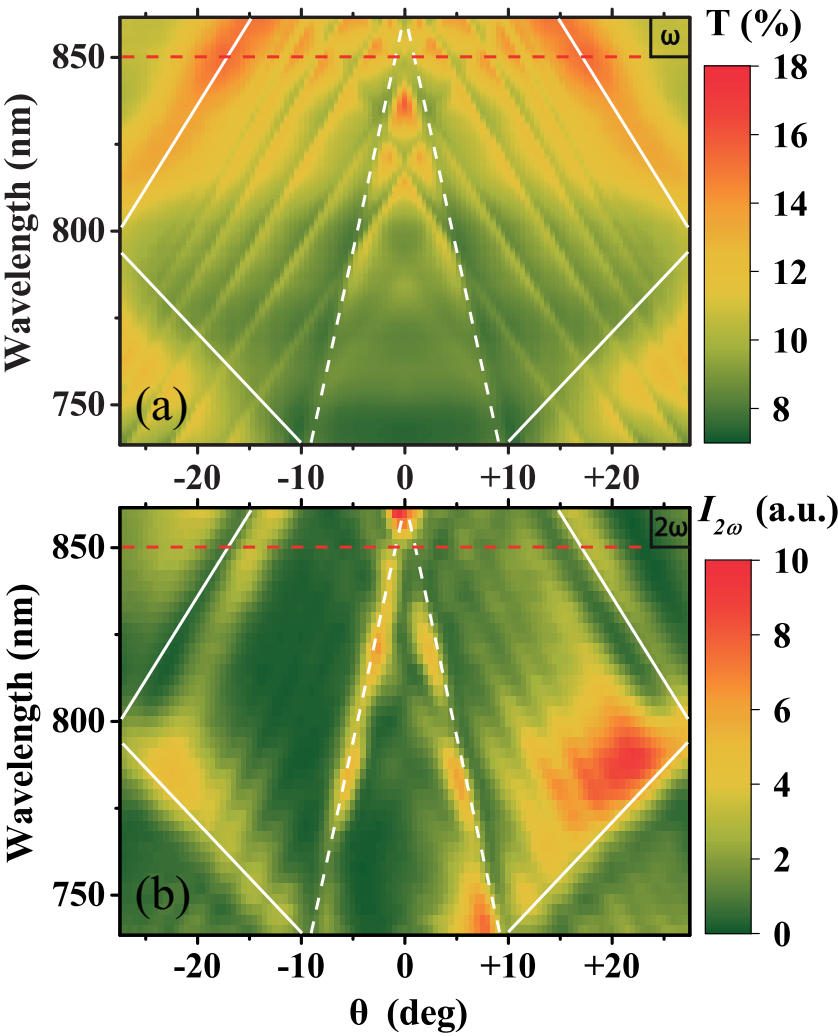

FIG. 2. Transmitted fundamental (a) and SHG (b) intensity versus the wavelength and angle of incidence. Solid and dashed lines show dispersion curves for, correspondingly, Au/BTIG and air/Au SPPs. Red dashed line indicates the analyzed cross section.

(shaded area), is replaced by a double maximum as the angle of incidence is reversed. We introduce the SHG angular contrast as $\rho_{\theta}=\left[I_{2 \omega}(+\theta)-I_{2 \omega}(-\theta)\right] /\left[I_{2 \omega}(+\theta)+I_{2 \omega}(-\theta)\right]$, where $I_{2 \omega}( \pm \theta) \propto\left|E_{2 \omega}( \pm \theta)\right|^{2}$ is the SHG intensity measured for $+\theta$ and $-\theta$ angles of incidence. Figure 3(b) shows the angular dependence of the $\rho_{\theta}$ at $850 \mathrm{~nm}$, demonstrating that the SPP excitation leads to a strong SHG angular asymmetry with a complex profile. It is important to note that the SHG output from a bare BTIG film also exhibits angular asymmetry, as is shown in the inset in Fig. 3(b). However, the angular dependencies of $\rho_{\theta}$ for the bare BTIG film and the plasmonic crystal differ drastically. The lack of SPP excitation severely limits the angular contrast values staying in this case below $30 \%$. In contrast, the SPP-driven $\rho_{\theta}$ reaches 95\% changing its sign three times in the vicinity of the resonance.

We emphasize that the considered plasmonic crystal supports the excitation of SPPs at the fundamental frequency $\omega$ only. As such, the spectral features in the nonlinear-optical response are attributed to the resonant effects experienced by the local amplitude and the local phase of the fundamental wave, as well as their variations, as the angle of incidence is reversed, $\theta \rightarrow-\theta$. We have performed the direct phase measurements of the SHG wave $e^{i \varphi}\left|E_{2 \omega}\right|$ by translating a reference nonlinear source (30-nm-thick ITO film) along the beam after the sample [36]. The interference of the SHG outputs of the sample and the reference source provides the 


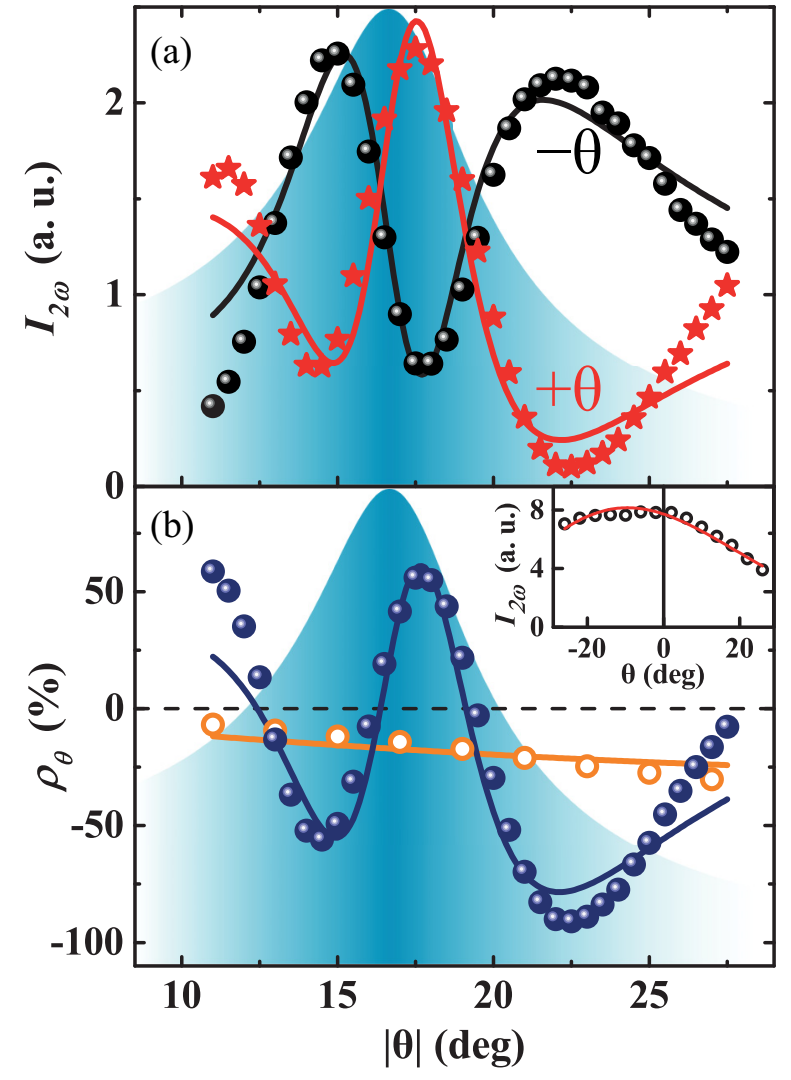

FIG. 3. (a) SHG intensity versus the absolute value of the incidence angle for positive (red stars) and negative (black spheres) $\theta$. (b) Angular contrast versus the absolute value of incidence angle for SHG from bare BTIG (orange empty circles) and plasmonic crystal (blue spheres). Solid curves are the fitting results. Blue shaded areas represent the Lorentzian profiles of the SPP electric field obtained from the fit using Eq. (4).

information about the phase of the SHG response. Figure 4 illustrates the angular spectra of the phase attained for the positive and negative $\theta$ values. The spectra have a classical steplike phase behavior with a $2 \pi$ shift, therefore we assume a Lorentzian shape of the resonant local field $E_{R}^{\omega}$. Using Eq. (1) with $k=\omega / c$ and the Au/BTIG-SPP refractive index $n=$ $\sqrt{\varepsilon_{\mathrm{Au}} \varepsilon_{\mathrm{BTIG}} /\left(\varepsilon_{\mathrm{Au}}+\varepsilon_{\mathrm{BTIG}}\right)} \approx 2.3[37,38]$, we get the following angular dependence of the local field:

$$
E_{R}^{\omega} \propto \frac{1}{f\left(\theta_{0}\right)^{2}-f(\theta)^{2}+2 i f(\theta) \gamma},
$$

with the resonant angle $\theta_{0}$, damping constant $\gamma$, and $f(\theta)=$ $1 /(n-|\sin \theta|)$.

Because of the SHG sentitivity to symmetry breaking and high absorption of BTIG at SHG wavelengths, we consider dipole SHG sources only at the interfaces. Consider the sample and laboratory coordinate frames introduced in Fig. 1 and denoted as $x z$ and $X Z$, respectively. The anisotropic BTIG (210) film has a point symmetry group $m$ with the mirror $(x z)$ plane. For the $p-\omega, p-2 \omega$ combination of polarizations $\left(E^{\omega}=E_{X}^{\omega}\right)$, we consider nonzero components of the surface susceptibility tensor $\hat{\chi}_{i j k}^{(2)}$ with $i, j, k=x, z$, which give rise to the SHG output.
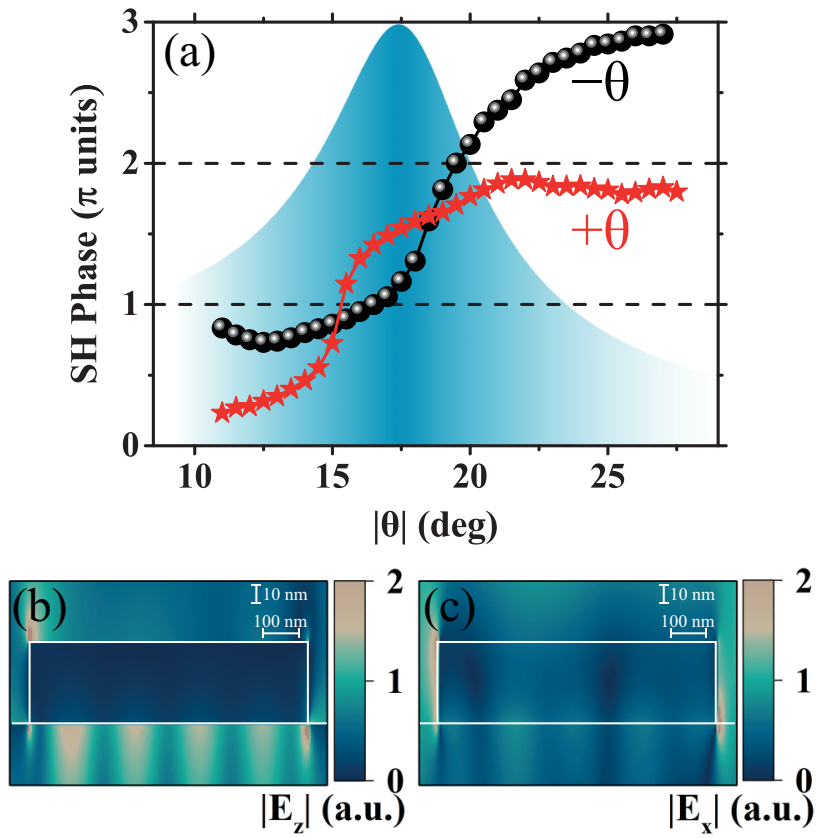

FIG. 4. (a) Phase of the SHG field for the fundamental wavelength $850 \mathrm{~nm}$ versus the absolute value of the incidence angle for positive (red stars) and negative (black spheres) $\theta$. Blue shaded area represents the Lorentzian profile of the SPP electric field obtained from the fit using Eq. (4). (b) $\left|E_{z}\right|$ and (c) $\left|E_{x}\right|$ distributions in the structure for the incidence angle of $17^{\circ}$.

When describing the angular SHG dependencies, we have to take into account the transformation rules for the nonlinear polarizations $P_{X}^{2 \omega}=P_{x}^{2 \omega} \cos \theta+P_{z}^{2 \omega} \sin \theta$ and the electric fields $E_{z}=E_{X} \sin \theta, E_{x}=E_{X} \cos \theta$. Therefore the SHG field induced by $\chi^{(2)}$ components with an odd number of $z$ subscripts $\left(\chi_{x x z}=\chi_{x z x} ; \chi_{z x x} ; \chi_{z z z}\right)$ will be odd in $\theta$, i.e., change their sign as the angle of incidence changes from $\theta$ to $-\theta$. On the contrary, the SHG field given by the $\chi^{(2)}$ components with an even number of $z$ subscripts such as $\chi_{x z z}$ do not change their sign upon reversing the angle $\theta$. The interference of the SHG fields induced by the $\chi^{(2)}$ components of different parity with respect to $\theta$ is thus responsible for the observed SHG angular asymmetry. Within this approach, the discussed effect is the result of an interplay of the SPP-enhanced local field effects and anisotropic SHG in the (210) BTIG film.

The SPPs excited at isotropic metal-air interface, which supports only odd $\chi^{(2)}$ components, produce no SHG asymmetry. Importantly, the very existence of the nonzero even $\chi^{(2)}$ components is inherent to the (210) BTIG film. This mechanism explains the asymmetry of the SHG response from a bare BTIG film, which is anisotropic with both even and odd $\chi^{(2)}$ components. For small incidence angles $\theta$ the in-plane field projections $E_{x}$ are dominant, and thus the SHG polarization $P_{X}^{2 \omega}$ can be described by two components of the $\hat{\chi}^{(2)}$ tensor: $\chi_{x x x}$ and $\chi_{z x x}$. In this case

$$
P_{X}^{2 \omega}(\theta)=\chi_{x x x} E_{x}^{2}(\theta) \cos \theta+\chi_{z x x} E_{x}^{2}(\theta) \sin ( \pm \theta),
$$

where $E_{x}(\theta)=E_{X} \cos \theta$. Equation (3) provides a good fit for both the SHG intensity $I^{2 \omega} \propto\left|P_{X}^{2 \omega}\right|^{2}$ and the angular contrast 
with $\chi^{(2)}$ components being the fit parameters, as shown in Fig. 3(b).

In the case of the plasmonic crystal, we consider two main SHG sources located at the air/Au (first) and Au/BTIG (second) interfaces (Fig. 1). The first interface is isotropic, while the second one has the symmetry of the BTIG allowing for the even $\chi^{(2)}$ components. Numerical simulations performed with the help of the designated LUMERICAL 8.0 software demonstrated that $E_{1 x}$ and $E_{1 z}$ at the Au/air interface are of the same order of magnitude [Figs. 4(b) and(c)]. In order to reduce the number of unknown fit parameters, we only consider the in-plane projection $E_{1 x}$. Taking into account both $E_{1 x}$ and $E_{1 z}$ could have slightly improved the quality of the model but would hardly be instructive. At the second (Au/BTIG) interface, the fundamental field is resonantly enhanced due to the SPP excitation, therefore we consider normal component of the electric field $E_{2 z}$ to be dominant [see Figs. 4(b and 4(c)]. Further, the fundamental field $E_{2 z}$ is a sum of the resonantly enhanced SPP field and the nonresonantly transmitted field: $E_{2 z}=E_{2 z}^{R}+E_{2 z}^{N R}$.

In order to illustrate the interference of even and odd contributions leading to large angular contrasts, we limit our model to just two terms with different parity with respect to $\theta$. Both SHG intensity and angular contrast [Figs. 3(a) and 3(b)] can be adequately described by these two terms originating from the $\chi_{1, z x x}$ and $\chi_{2, x z z}$ components:

$$
\begin{aligned}
P_{X}^{2 \omega}(\theta)= & \chi_{2, x z z}\left[E_{2 z}^{R}(\theta)+E_{2 z}^{N R}\right]^{2} \cos \theta \\
& +\chi_{1, z x x} E_{1 x}^{2} \sin ( \pm \theta),
\end{aligned}
$$

where the resonant SPP-assisted local field at the fundamental wavelength $E_{2 z}^{R}(\theta)$ is given by Eq. (2). The fitting of experimental data [Fig. 2(a)] provides $\theta_{0}=(17.0 \pm 0.2)^{\circ}$ and $\gamma=(0.012 \pm 0.001)$.

We note that this asymmetry can be also observed in the linear response [compare to Fig. 2(b)] if the BTIG film is magnetized along the $y$ axis. The magnetization $M_{y}$ will induce nondiagonal components of the susceptibility tensor $\varepsilon_{x z} \propto M_{y}$ of the dielectric film and thus change the Au/BTIG SPP dispersion [5,39-41]. Again, since this shift is induced by a component with an odd number of $z$ subscripts, it will be odd with respect to the SPP propagation direction and thus to $\theta$. However, the corresponding change in the transmittance (angular contrast) is proportional to the gyration vector of BTIG and is therefore relatively small (about $0.1 \%-1 \%$ ). In the nonlinear optics, the observed magneto-optical SPP-induced effects are rarely discussed along these lines [19,22-25,42,43] due to the complexity of the nonlinear-optical output. However, the nonlinear counterpart of this effect together with large values of the asymmetry-driven SHG output modulation (up to 95\%) indicates high prospects for nonlinear active magnetoplasmonics $[43,44]$. Successful engineering of such systems with an appropriate optical response is likely to realize active plasmonic structures with a magnetic field as one of the convenient control knobs.

Summing up, we demonstrate a strong anisotropy-driven asymmetry effect in the SHG output from a Au/BTIG plasmonic crystal. The SHG asymmetry is evidenced by the measurement of SHG intensity versus the wavelength and the angle of incidence. As compared with a bare BTIG film, the angular SHG asymmetry is strongly modulated and enhanced due to the SPP excitation. We argue that the SHG asymmetry originates from the intrinsic anisotropy of the Au/BTIG interface. Using simple symmetry considerations for the two SHG sources at the interfaces, we show that the SHG angular asymmetry is driven by an interplay between the contributions with different parity with respect to the angle of incidence. Our results visualize a new effect in nonlinear plasmonics and demonstrate the broad opportunities provided by SPPs at anisotropic interfaces.

The authors would like to thank Dr. A. Melnikov, Dr. T. Kampfrath, and Dr. K. Campen for fruitful and enlightening discussions and Dr. S. Semin for the technical assistance with the experiments.
[1] M. Kuwata-Gonokami, N. Saito, Y. Ino, M. Kauranen, K. Jefimovs, T. Vallius, J. Turunen, and Y. Svirko, Phys. Rev. Lett. 95, 227401 (2005).

[2] H. J. Lezec, J. A. Dionne, and H. A. Atwater, Science 316, 430 (2007).

[3] V. V. Temnov, G. Armelles, U. Woggon, D. Guzatov, A. Cebollada, A. Garcia-Martin, J. M. Garcia-Martin, T. Thomay, A. Leitenstorfer, and R. Bratschitsch, Nat. Photonics 4, 107 (2010).

[4] P. Berini and I. De Leon, Nat. Photonics 6, 16 (2012).

[5] V. I. Belotelov, L. E. Kreilkamp, I. A. Akimov, A. N. Kalish, D. A. Bykov, S. Kasture, V. J. Yallapragada, A. V. Gopal, A. M. Grishin, S. I. Khartsev, M. Nur-E-Alam, M. Vasiliev, L. L. Doskolovich, D. R. Yakovlev, K. Alameh, A. K. Zvezdin, and M. Bayer, Nat. Commun. 4, 2128 (2013).

[6] V. V. Temnov, C. Klieber, K. A. Nelson, T. Thomay, V. Knittel, A. Leitenstorfer, D. Makarov, M. Albrecht, and R. Bratschitsch, Nat. Commun. 4, 1468 (2013).
[7] J.-M. Yi, A. Cuche, E. Devaux, C. Genet, and T. W. Ebbesen, ACS Photonics 1, 365 (2014).

[8] J. J. Foley IV, J. M. McMahon, G. C. Schatz, H. Harutyunyan, G. P. Wiederrecht, and S. K. Gray, ACS Photonics 1, 739 (2014).

[9] L. Lin, M. Zapata, M. Xiong, Z. Liu, S. Wang, H. Xu, A. G. Borisov, H. Gu, P. Nordlander, J. Aizpurua, and J. Ye, Nano Lett. 15, 6419 (2015).

[10] A. Lauchner, A. E. Schlather, A. Manjavacas, Y. Cui, M. J. McClain, G. J. Stec, F. J. García de Abajo, P. Nordlander, and N. J. Halas, Nano Lett. 15, 6208 (2015).

[11] H. J. Simon, D. E. Michell, and J. G. Watson, Phys. Rev. Lett. 33, 1531 (1974).

[12] F. De Martini and Y. R. Shen, Phys. Rev. Lett. 36, 216 (1976).

[13] M. G. Albrecht and J. A. Creighton, J. Am. Chem. Soc. 99, 5215 (1977).

[14] I. Gontijo, M. Boroditsky, E. Yablonovitch, S. Keller, U. K. Mishra, and S. P. DenBaars, Phys. Rev. B 60, 11564 (1999). 
[15] S. Palomba and L. Novotny, Phys. Rev. Lett. 101, 056802 (2008).

[16] V. K. Valev, A. V. Silhanek, N. Verellen, W. Gillijns, P. Van Dorpe, O. A. Aktsipetrov, G. A. E. Vandenbosch, V. V. Moshchalkov, and T. Verbiest, Phys. Rev. Lett. 104, 127401 (2010).

[17] T. Utikal, T. Zentgraf, T. Paul, C. Rockstuhl, F. Lederer, M. Lippitz, and H. Giessen, Phys. Rev. Lett. 106, 133901 (2011).

[18] N. B. Grosse, J. Heckmann, and U. Woggon, Phys. Rev. Lett. 108, 136802 (2012).

[19] V. L. Krutyanskiy, I. A. Kolmychek, E. A. Gan'shina, T. V. Murzina, P. Evans, R. Pollard, A. A. Stashkevich, G. A. Wurtz, and A. V. Zayats, Phys. Rev. B 87, 035116 (2013).

[20] I. Razdolski, S. Parchenko, A. Stupakiewicz, S. Semin, A. Stognij, A. Maziewski, A. Kirilyuk, and T. Rasing, ACS Photonics 2, 20 (2015).

[21] M. Kauranen and A. V. Zayats, Nat. Photonics 6, 737 (2012).

[22] I. Razdolski, D. G. Gheorghe, E. Melander, B. Hjörvarsson, P. Patoka, A. V. Kimel, A. Kirilyuk, E. Th. Papaioannou, and Th. Rasing, Phys. Rev. B 88, 075436 (2013).

[23] W. Zheng, A. T. Hanbicki, B. T. Jonker, and G. Lüpke, Sci. Rep. 4, 6191 (2014).

[24] V. L. Krutyanskiy, A. L. Chekhov, V. A. Ketsko, A. I. Stognij, and T. V. Murzina, Phys. Rev. B 91, 121411 (2015).

[25] V. Pavlov, G. Tessier, C. Malouin, P. Georges, A. Brun, D. Renard, P. Meyer, J. Ferré, and P. Beauvillain, Appl. Phys. Lett. 75, 190 (1999).

[26] T. Verbiest, M. Kauranen, Y. V. Van Rompaey, and A. Persoons, Phys. Rev. Lett. 77, 1456 (1996).

[27] M. Liscidini and J. E. Sipe, Phys. Rev. B 81, 115335 (2010).

[28] L. E. Kreilkamp, V. I. Belotelov, J. Y. Chin, S. Neutzner, D. Dregely, T. Wehlus, I. A. Akimov, M. Bayer, B. Stritzker, and H. Giessen, Phys. Rev. X 3, 041019 (2013).
[29] M. Pohl, L. E. Kreilkamp, V. I. Belotelov, I. A. Akimov, A. N. Kalish, N. E. Khokhlov, V. J. Yallapragada, A. V. Gopal, M. Nur-E-Alam, M. Vasiliev, D. R. Yakovlev, K. Alameh, A. K. Zvezdin, and M. Bayer, New J. Phys. 15, 075024 (2013).

[30] A. Chekhov, V. Krutyanskiy, A. Shaimanov, A. Stognij, and T. Murzina, Opt. Express 22, 17762 (2014).

[31] A. L. Chekhov, V. L. Krutyanskiy, V. A. Ketsko, A. I. Stognij, and T. V. Murzina, Opt. Mater. Express 5, 1647 (2015).

[32] H. Raether, Surface Plasmons on Smooth and Rough Surfaces and on Gratings (Springer, Berlin, 1988).

[33] M. Siltanen, S. Leivo, P. Voima, M. Kauranen, P. Karvinen, P. Vahimaa, and M. Kuittinen, Appl. Phys. Lett. 91, 111109 (2007).

[34] B. Luk'yanchuk, N. I. Zheludev, S. A. Maier, N. J. Halas, P. Nordlander, H. Giessen, and C. T. Chong, Nat. Mater. 9, 707 (2010).

[35] T. W. Ebbesen, H. J. Lezec, H. F. Ghaemi, T. Thio, and P. A. Wolff, Nature (London) 391, 667 (1998).

[36] R. Stolle, G. Marowsky, E. Schwarzberg, and G. Berkovic, Appl. Phys. B 63, 491 (1996).

[37] P. B. Johnson and R. W. Christy, Phys. Rev. B 6, 4370 (1972).

[38] V. Doormann, J.-P. Krumme, C.-P. Klages, and M. Erman, Appl. Phys. A 34, 223 (1984).

[39] A. K. Zvezdin and V. A. Kotov, Modern Magnetooptics and Magnetooptical Materials (Taylor \& Francis, London, 1997).

[40] M. S. Kushwaha and P. Halevi, Phys. Rev. B 36, 5960 (1987).

[41] A. A. Grunin, A. G. Zhdanov, A. A. Ezhov, E. A. Ganshina, and A. A. Fedyanin, Appl. Phys. Lett. 97, 261908 (2010).

[42] W. Zheng, A. T. Hanbicki, B. T. Jonker, and G. Lüpke, Opt. Express 21, 28842 (2013).

[43] I. Razdolski, D. Makarov, O. G. Schmidt, A. Kirilyuk, T. Rasing, and V. V. Temnov, ACS Photonics 3, 179 (2016).

[44] W. Zheng, X. Liu, A. T. Hanbicki, B. T. Jonker, and G. Lüpke, Opt. Mater. Express 5, 2597 (2015). 